\title{
Situación de salud en una comunidad afrocolombiana sobreviviente a la masacre de Bojayá, Chocó
}

\author{
Health Status within an Afro-Colombian Community Survivor of the Bojayá \\ Massacre, Chocó
}

\author{
Situação de saúde em uma comunidade afro colombiana sobrevivente ao \\ massacre de Bojayá, Chocó
}

Zulma Consuelo Urrego-Mendoza'; Germán Piñeros-Cortés²; Natalia Moreno-Arévalo3; Mario Esteban Hernández-Álvarez"; María Clara Calderón-Scioville5; Daisy Mariana Moreno Martínez;; María Alejandra Rojas-Ordoñez;; Jonathan Alexánder Peralta-Jiménez .

1 Médica cirujana, PhD. Salud Pública. Universidad Nacional de Colombia. Bogotá, Colombia. zulcurregom@unal.edu.co; https://orcid. org/0000-0003-1732-4725

2 Antropólogo, Universidad Nacional de Colombia. Bogotá, Colombia. gpinerosc@unal.edu.co; https://orcid.org/0000-0001-9353-1617

3 Bacterióloga y laboratorista clínica. Universidad Nacional de Colombia, Bogotá Colombia. namorenoa@unal.edu.co; https://orcid. org/0000-0002-6540-6517

4 Médico, PhD. Historia. Universidad Nacional de Colombia. Bogotá, Colombia. mehernandeza@unal.edu.co; https://orcid.org/00000002-3996-7337

5 Psicóloga, especialista en Intervención Sistémica de la Familia. Universidad Nacional de Colombia. Bogotá, Colombia. mccscioville@ yahoo.es; https://orcid.org/0000-0002-7709-8662

6 Fonoaudióloga. Universidad Nacional de Colombia. Bogotá, Colombia. dammorenoma@unal.edu.co; https://orcid.org/0000-0003$1753-1230$

7 Fisioterapeuta, MgSc. Salud Pública, Universidad Nacional de Colombia. Bogotá, Colombia. maarojasor@unal.edu.co; https://orcid. org/0000-0001-9269-3470

$8 \quad$ Médico cirujano. Universidad Nacional de Colombia, Bogotá, Colombia. japeraltaj@unal.edu.co; https://orcid.org/0000-0002-34196879

Recibido: 19/12/2019. Aprobado: 18/12/2020. Publicado: 26/01/2021

Urrego-Mendoza ZC, Piñeros-Cortés G, Moreno-Arévalo N, Hernández-Álvarez ME, Calderón-Scioville MC, Moreno Martínez DM, Rojas-Ordoñez MA, Peralta-Jiménez JA. Situación de salud en una comunidad afrocolombiana sobreviviente a la masacre de Bojayá, Chocó. Rev. Fac. Nac. Salud Pública. 2021;39(1):e340623. DOI: https://doi.org/10.17533/udea.rfnsp.e340623

\section{Resumen}

Objetivo: En el marco del proyecto de extensión solidaria universitaria "Laboratorio de salud rural e intercultural comunidad de Bojayá, Chocó", este artículo presenta los resultados correspondientes a la fase de diagnóstico individual y la aproximación inicial al diagnóstico colectivo de la situación de salud presente en un colectivo afrocolombiano sobreviviente a una masacre emblemática ocurrida en Colombia. Metodología: Exploración preliminar y elementos de diagnóstico comunitario con 66 sobrevivientes de la masacre del 2 de mayo de 2002 en el poblado de Bellavista, en el municipio de Bojayá, Chocó. Resultados: Se identificaron condiciones socioeconómicas deficientes, diagnósticos 
clínicos desatendidos, y se efectuó la aproximación inicial a potencialidades disponibles para el afrontamiento de las necesidades en salud individuales y colectivas de la población abordada, representadas en saberes comunitarios y posibilidad de acceso a programas y servicios institucionales en salud para víctimas. Conclusiones: Pese a que transcurrieron más de 15 años desde el momento de ocurrencia de la masacre de Bojayá hasta nuestro trabajo con la comunidad, se encontró persistencia de múltiples necesidades insatisfechas que afectan negativamente la situación de salud individual y colectiva, sin adecuada ni oportuna intervención. Cualquier acción futura que se plantee para atender tales asuntos debe tomar en cuenta las condiciones histórico-sociales que configuran los procesos de salud-enfermedad-cuidado en dicha comunidad.

-Palabras clave: Diagnóstico de la situación de salud en grupos específicos; conflictos armados; grupo de ascendencia continental africana; salud rural; salud pública.

\section{Abstract}

Objective: Within the framework of the project of solidary continuing education "Laboratory of rural and intercultural health community of Bojayá, Chocó", this paper presents the results of the individual diagnostic phase and the initial approach to the collective diagnosis of the health status diagnosis presented in an Afro-Colombian group surviving to a notorious massacre occurred in Colombia. Methodology: Preliminary exploration and community diagnosis elements with 66 survivors of the massacre occurred on May 2, 2002 in the Bellavista village, in the municipality of Bojayá, Chocó. Results: Deficient socio-economic conditions and neglected clinical diagnosis were identified. An initial approach to the available potentialities to face the individual and collective health needs of the approached population was made, represented by community knowledge and accessibility to institutional programs and health services for victims. Conclusions: Although more than 15 years have passed from the occurrence of the Bojayá massacre until our work with the community began, the persistence of numerous unsatisfied needs that negatively affect the individual and collective health status, without adequate and timely intervention, was found. Any further action looking to address such matters should take into account the socio-historical conditions that define the health-illness-care process in that community.

---------Keywords: diagnosis of the health status in specific groups, armed conflicts, African continental ancestry group, rural health, public health.

\section{Resumo}

Objetivo: No âmbito do projeto de extensão solidária universitária "Laboratório de saúde rural e intercultural comunidade de Bojayá, Chocó", este artigo apresenta os resultados correspondentes à fase de diagnóstico individual e a aproximação inicial ao diagnóstico coletivo da situação de saúde presente num coletivo afro colombiano sobrevivente a um massacre emblemático ocorrido na Colômbia. Metodologia: Exploração preliminar e elementos de diagnóstico comunitário com 66 sobreviventes do massacre de 2 de maio de 2002 no povoado de Bellavista, no município de Bojayá, Chocó. Resultados: Foram identificadas condições socioeconômicas deficientes, diagnósticos clínicos negligenciados e efetuouse a aproximação inicial a disponíveis potencialidades para o enfrentamento das necessidades em saúde individuais e

\section{Introducción}

Bojayá, en el departamento de Chocó, sobre el río Atrato, es uno de los municipios priorizados para la implementación de los "Programas de desarrollo con enfoque territorial", establecidos en el Acuerdo Final de Paz firmado entre las Fuerzas Armadas Revolucionarias de Colombia - Ejército del Pueblo (FARC-EP) y el Estado Colombiano en 2016 [1]. Ello genera un escenario favo- coletivas da população abordada, representadas em saberes comunitários e possibilidade de acesso a programas e serviços institucionais em saúde para vítimas. Conclusões: Apesar do fato de que se passaram 15 anos do acontecimento do massacre de Bojayá até o nosso trabalho com a comunidade, foi encontrada a persistência de múltiplas necessidades insatisfeitas que afetam de forma negativa a situação de saúde individual e coletiva, sem intervenção adequada e oportuna. Qualquer ação futura planejada para atender tais assuntos, deve considerar as condições histórico-sociais que caracterizam os processos de saúde-doença-cuidado em tal comunidade

-Palavras-chave: Diagnóstico da Situação de Saúde em Grupos Específicos; Conflitos Armados; Grupo com Ancestrais do Continente Africano; Saúde da População Rural; Saúde Pública.

rable para desarrollar iniciativas que articulen procesos de movilización social de larga data, con la voluntad política e inversión presupuestal que se desprende de la implementación de las diferentes medidas trazadas desde el acuerdo de paz. A este propósito, la universidad pública se ha unido mediante múltiples enfoques y acciones. No obstante, este entorno también genera retos importantes, asociados a la multiplicidad de actores interesados en hacer presencia en el territorio, pues esto representa una 
amenaza para los procesos organizativos comunitarios, lo que propicia el desarrollo de iniciativas no siempre sostenibles ni articuladas a las demandas estructurales de la comunidad.

Además, las iniciativas en torno a un proceso de paz con las FARC-EP y el Ejército de Liberación Nacional abrieron una ventana de oportunidad para lograr transformaciones profundas, pese a las dificultades posteriores para su implementación. Asimismo, el logro de una paz "estable y duradera" requiere la transformación de las condiciones que han generado la guerra: las inequidades, el modelo económico y un sistema político cerrado. Se entiende que la dinámica social, política y económica en los "territorios de paz" es la punta de lanza de transformaciones más profundas de la relación entre lo urbano y lo rural, y de la institucionalidad pública, por lo cual la perspectiva territorial incluida en el Acuerdo Final permite articular la salud a múltiples procesos sociales locales de superación de inequidades y de cambio en la estructura del poder social.

Así, en el marco del proyecto de extensión solidaria universitaria "Laboratorio de salud rural e intercultural comunidad de Bojayá, Chocó", que pretende desarrollar, en fases sucesivas, conocimiento sobre necesidades individuales y colectivas en salud, recursos individuales y colectivos para el afrontamiento, así como líneas de trabajo posibles para el diseño y la operación de estrategias de intervención aplicables en un territorio afectado crónicamente por el conflicto armado interno como el de Bojayá, este artículo presenta los resultados correspondientes a la fase de diagnóstico individual y la aproximación inicial al diagnóstico colectivo de la situación de salud presente en un colectivo afrocolombiano sobreviviente a una masacre emblemática ocurrida en Colombia.

De manera específica, se buscó, en primer lugar, caracterizar, desde una perspectiva sociodemográfica, al grupo de esas personas direccionadas, actualmente residentes en Bellavista, municipio de Bojayá, y en el municipio de Quibdó, Chocó; en segundo lugar, elaborar el diagnóstico de sus necesidades en salud individual desde la perspectiva de un equipo interdisciplinario de profesionales en salud, y en tercer lugar, realizar una aproximación inicial al diagnóstico de salud colectiva, con participación de la comunidad.

\section{Metodología}

El método seguido correspondió al de una exploración preliminar y de diagnóstico comunitario, que incluye evaluaciones diagnósticas clínicas individuales, con 66 sobrevivientes de la masacre del 2 de mayo de 2002 en Bellavista (Bojayá, Chocó), quienes fueron direccionados hacia el proyecto de extensión solidaria universitaria a partir de la organización comunitaria Comité por los Derechos de las Víctimas de la Masacre de Bojayá.

Según registros del Comité, el total de sobrevivientes directos de la masacre está constituido por 157 personas que habitan en múltiples localizaciones de Colombia. La población objetivo de la intervención es de 141 personas sobrevivientes de la masacre, sin incluir a quienes viven actualmente en departamentos y municipios distantes del sitio donde ocurrió el evento índice. El mismo Comité priorizó un grupo de 66 personas para las actividades que se reseñan, con quienes se trabajó en los componentes colectivos e individuales planteados para esta fase del proyecto (en Bojayá: 35 personas, y en Quibdó: 31 personas) [2]; además de ellos, otras 27 personas pertenecientes a la misma comunidad se sumaron voluntariamente a las actividades colectivas.

Para la aproximación inicial a las necesidades individuales en salud, cada una de las áreas del equipo interdisciplinario participante (fonoaudiología, fisioterapia, salud pública, psicología-psiquiatría, medicina general) efectuó una entrevista, con valoración clínica e intervención inicial, mediante educación en salud, e intervención terapéutica de sesión única desde el área de salud mental. La evaluación tomó entre 40 y 120 minutos por área, por persona evaluada.

Para la aproximación inicial a las necesidades y potencialidades colectivas en salud, las áreas de antropología y salud pública, junto con líderes comunitarios, efectuaron grupos conversacionales no dirigidos, desarrollados durante el tiempo de espera en la atención individual.

Estas actividades estuvieron enmarcadas temporalmente en un período de dos meses, desde su planificación hasta la construcción de informes del trabajo de campo; y se acompañaron de otras actividades (que no son presentadas a profundidad en este texto), cuya duración total de inicio a fin fue de 12 meses.

La totalidad de las actividades desarrolladas durante ese año, y que contextualizan lo presentado en el artículo, se enlistan a continuación:

- Formulación inicial de la estrategia de entrada al campo, en coordinación con líder comunitaria por parte de las áreas de antropología y salud pública, con apoyo en referentes teóricos útiles a ambas disciplinas [3-6].

- Construcción de un estado del arte, por antropología y salud pública.

- Construcción de la propuesta de trabajo en campo, por parte de un equipo interdisciplinario (antropología, fisioterapia, fonoaudiología, líderes comunitarios, medicina general, psiquiatría, psicología, salud pública).

- Evaluación individual de necesidades y potencialidades en salud, por equipo interdisciplinario. 
- Exploración preliminar colectiva de necesidades y potencialidades comunitarias en salud, por equipo interdisciplinario.

- Taller de primeros auxilios psicológicos, dirigido por el área de psicología y orientado a un grupo de líderes comunitarios e institucionales ubicados en Bellavista.

- Retroalimentación a la comunidad participante.

- Construcción de informes y productos finales.

Lo anterior se llevó a cabo en el marco de una actividad solidaria de extensión universitaria. Por tratarse de una población vulnerable, fueron tomados consentimientos informados individuales para la realización de los procedimientos de evaluación diagnóstica clínica, para la toma de registros de audio y video, y para la difusión de productos derivados en contextos académicos. Igualmente, se solicitó aval comunitario para la realización de la intervención.

El proyecto fue aprobado por el comité evaluador de propuestas constituido para la Convocatoria Nacional de Extensión Solidaria 2017 de la Universidad Nacional de Colombia.

Para la protección de la integridad del equipo de trabajo, se cumplió con todos los protocolos para salida de campo requeridos por nuestra institución, según lo establecido a partir de la Resolución 006 de 2010 del Consejo de Bienestar Universitario de la Universidad Nacional de Colombia.

Los datos tomados a partir de los registros de la práctica fueron procesados en Excel ${ }$ 2016, mediante estadística descriptiva para lo cuantitativo, y por análisis narrativo manual, mediado por matrices, para lo cualitativo.

\section{Resultados}

El artículo se centra en presentar los resultados de las evaluaciones individuales de necesidades y potencialidades en salud, así como el resultado a la aproximación inicial a las necesidades y potencialidades comunitarias en salud.

\section{Caracterización sociodemográfica}

Las personas participantes fueron mayoritariamente mujeres (44 personas; $67 \%$ ), con edades más frecuentes entre los 27 y 59 años (46 personas; $70 \%$ ).

En cuanto al nivel educativo, la mayoría (15 personas; $23 \%$ ) alcanzó la secundaria, pero no la culminó. A raíz del desplazamiento forzado, muchas de las personas más jóvenes dejaron sus estudios y se dedicaron a trabajar, para su subsistencia.

Diagnóstico de salud pública a partir de componentes individuales y colectivos
Según los resultados de las entrevistas individuales, en los dos municipios evaluados se evidencian problemas de salud asociados con la masacre, que no han recibido un adecuado y oportuno tratamiento. Esto está relacionado con la existencia de barreras de acceso a los servicios de salud.

Para Quibdó, las barreras geográficas, económicas $y$ administrativas (36 personas; $55 \%$ ), identificadas por la población como un conjunto, son las más comunes. Lo anterior, debido a que los lugares donde se encuentran las instituciones prestadoras de salud son lejanos de sus residencias, sumado a un transporte precario y costoso. Igualmente, no existe un trabajo formal que permita tener recursos para los gastos de bolsillo que implica la atención médica, y se registran dificultades o demoras para la autorización y el acceso efectivo a consultas, exámenes especializados o medicamentos.

En cuanto a los habitantes en Bellavista (Bojayá), las barreras administrativas fueron las más frecuentes (32 personas; $48 \%$ ), por razones similares a las ya descritas para Quibdó; no obstante, también experimentaron importantes barreras geográficas, económicas y culturales. Los desplazados a la ciudad de Quibdó enfrentan mayores dificultades laborales, pues carecen de trabajos estables que aseguren la manutención familiar.

Por otro lado, la intermitencia del servicio del agua potable y la ausencia de alcantarillado adecuado en ambos lugares facilita la presencia de recurrentes infecciones parasitarias y de piel, sin que hasta el momento el Gobierno haya atendido la exigencia de este derecho al saneamiento básico como parte integrante del derecho a la salud. Lo anterior responde no solo a las condiciones de vivienda mencionadas, sino también a la precaria formación educativa y, en consecuencia, a una fuente de ingresos insuficiente para la satisfacción de sus necesidades básicas en este aspecto.

El diagnóstico colectivo preliminar elaborado desde las áreas de antropología y salud pública en los municipios intervenidos identificó enormes necesidades insatisfechas en torno a condiciones dignas para la vida. Como mayores percepciones de requerimientos de atención en salud para la comunidad, obtenidas en los grupos conversacionales, estuvieron las molestias oculares, las esquirlas que permanecen no atendidas dentro del cuerpo, los problemas de fertilidad en las mujeres, y el incremento percibido en la mortalidad por cáncer entre las personas sobrevivientes a la masacre.

Quedó, además, en evidencia, la necesidad de una indagación más profunda sobre los mecanismos de reproducción social del conocimiento tradicional en salud: quiénes y por qué lo detentan, desde qué edad deben ser enseñados y, en una instancia más íntima, cómo son esos espacios de enseñanza y cómo se va introduciendo al aprendiz de médico tradicional en las prácticas más complejas. Esto, debido a la importancia de la red de 
sabedores ancestrales (hombres y mujeres) en el cuidado de la salud, asociados a la etnobotánica y empleados en la fitoterapia, sin dejar a un lado las prácticas culturales, como los alabaos, " utilizadas como estrategias de afrontamiento y sanación psicosocial.

\section{Diagnóstico de salud física y mental individual}

En el campo de la salud física, evaluada desde el área de medicina general, prevalecieron los siguientes síntomas por sistemas: nervioso — cefaleas $(n=55 / 66$ personas; $83 \%$ ) y parestesias (23/66 personas; $35 \%$ ) -; osteomuscular - dolor lumbar o articular (43/66 personas; $65 \%$ ) - ; sentidos - alteraciones visuales (43/66 personas; $65 \%$ y percepción de hipoacusia o tinnitus $(60 / 66$ personas; $91 \%$ - -

Los principales diagnósticos, de manera general, fueron: osteoartrosis (38/66 personas; $58 \%$ ), hernias discales (30/66 personas; $46 \%$ ), migraña (28/66 personas; $42 \%$ ), hipertensión arterial crónica (26/66 personas; $39 \%)$, otitis media ( $21 / 66$ personas; $32 \%$ ) y cuerpo extraño alojado (22/66 personas; $33 \%$ ). Todas las personas recibieron educación en salud, según su caso.

En lo que respecta a las evaluaciones por fisioterapia, en su totalidad las 66 personas evaluadas (100\%) tuvieron hallazgos positivos que requieren intervención diagnóstica o terapéutica por condiciones médicas que afectan su funcionalidad (artritis o artrosis, Parkinson, cicatrices y otras secuelas de trauma físico). El dolor crónico fue frecuente. Asimismo, se encontró que presentan alteraciones posturales, bajo acondicionamiento físico u obesidad, que generan otros síntomas.

También se identificaron transformaciones en el esquema y la consciencia corporal, así como en la corporalidad, a raíz de los sucesos victimizantes, pero ello debe ser objeto de profundización futura.

Respecto a la salud auditiva, las valoraciones por fonoaudiología, mediante otoscopia, timpanometría, reflejos ipsilaterales, promedio de tonos puros, audiometría y logoaudiometría, comprobaron presencia de hipoacusia en $43 / 66$ personas evaluadas ( $65 \%$ ). En todos los casos, se brindó educación en actividad física y en salud auditiva.

En cuanto a la salud mental, los principales síntomas detectados fueron de tipo postraumático (39/66 personas; $59 \%$ ) y 12 personas presentaron síntomas relacionados con depresión (18\%). En concordancia, el diagnóstico clínico que ocupó el primer lugar fue el trastorno por estrés postraumático (37/66 personas; $56 \%$ ), seguido por los trastornos depresivos mayores ( $8 / 66$ personas; $12 \%$ ). Los duelos no resueltos, y complicados por sintomatología postraumática y depresiva, fueron la norma.

En todos los casos, además de la valoración clínica, se brindó una intervención terapéutica de sesión única, ${ }^{\dagger}$ por profesionales en salud mental, con el debido entrenamiento en la técnica, y se direccionó a las personas hacia sus correspondientes servicios de salud, para solicitar inicio de atención terapéutica adicional cuando así lo requerían.

Vale resaltar que fueron evidentes las capacidades de afrontamiento exhibidas por los bojayaceños sobrevivientes de la masacre y las múltiples manifestaciones de resiliencia que les han permitido sobrellevar su situación hasta la fecha, reinventando la vida pese a la adversidad.

\section{Discusión}

La región del Pacífico colombiano, habitada por una mayoría de origen afro e indígena, ha sido desde su incorporación al imperio español, en el siglo XVII, una región de conflicto. Dadas sus características naturales y abundantes recursos naturales y mineros [9], además de su privilegiada posición con fácil acceso a los océanos Atlántico y Pacífico, es uno de los lugares del país donde la violencia se ha manifestado y naturalizado de forma más persistente [10], e irónicamente, una de las que más ha padecido del abandono estatal, generando un escenario propicio para la guerra abierta entre grupos armados legales e ilegales por el control general del mencionado territorio, especialmente en el departamento de Chocó. Esto se evidencia en los resultados que por componente presentamos en líneas anteriores, con especial enfoque en las condiciones de vida de la población objetivo.

Luego de la masacre, múltiples intervenciones desde diversas ciencias y experticias se orientaron para ofrecer asesorías y ayudas en servicios básicos, como salud y educación, áreas históricamente abandonadas desde la soberanía estatal, con el fin de contribuir a la reconstrucción del tejido social y la reparación integral de las víctimas [11-14]. No obstante, la salud de las víctimas permanece con múltiples necesidades insatisfechas hasta la actualidad [15].

* Los alabaos son cantos de alabanza y fúnebres, propios de la tradición afrocolombiana en la región del Pacífico.

$\dagger \quad$ La intervención terapéutica de sesión única es una modalidad de intervención psicologica que ha sido probada y recomendada en Colombia por algunas organizaciones internacionales de ayuda humanitaria en salud, como alternativa para personas afectadas por el conflicto armado, habitantes en zonas rurales, donde no es posible garantizar atención terapéutica convencional con continuidad por parte de un mismo terapeuta o equipo de trabajo, dadas las dificultades de acceso geografico o por dinamicas del conficto. Diseñada inicialmente por el psiquiatra Moshe Talmon, esta intervención psicoterapeutica especializada ha sido descrita con resultados favorables por múltiples investigadores internacionales, en diversas poblaciones $[7,8]$. 
Últimamente, la escasa implementación del acuerdo de paz no ha permitido tampoco la transformación de las condiciones de base que mantienen esa precaria situación de salud individual y colectiva; tampoco ha permitido brindar soluciones de corto plazo ante las necesidades percibidas y emergentes en salud, y en otros ámbitos de la vida indispensables para vivir bien.

Como áreas que permanecen sin debida satisfacción, resalta todo aquello que se refiere a la reconstrucción de la memoria histórica de la masacre desde perspectivas propias y externas [13,16-20], la reconstrucción del tejido social [16,21], y el adecuado afrontamiento de los procesos de desterritorialización [22], retorno, reubicación, reterritorialización y abordaje de las transformaciones socioculturales emergentes tanto en el municipio [11,16, 23-26] como en otras áreas del país a donde han llegado comunidades étnicas, afrocolombianas e indígenas, desplazadas por la violencia en general [27], y por la derivada de la masacre de Bojayá en particular.

Lo anterior, considerando que la evidencia durante esta fase inicial y exploratoria mostró que las deficiencias en estos aspectos, incluyendo la atención en salud, se vienen manifestando incluso desde tiempos previos al hecho victimizante índice que nos ocupa.

Cabe señalar que las iniciativas desde la sociedad civil han suplido algunas de las necesidades de reparación colectiva. Ejemplo de ello es el grupo "Cantadoras de Pogue". Así, el canto, como elemento del ritmo y como conocimiento ancestral, es, a su vez, un mecanismo de reparación social [28], sustentando la construcción de la memoria histórica, la tan necesaria reparación y la denuncia de los daños causados por la guerra, todo en consonancia con la espiritualidad afrochocoana [17]. De este modo, sus intérpretes, las mujeres, se han ubicado como sujetos políticos activos y contestatarios a través de esta manifestación cultural [29]. Lo anterior fue evidenciado durante las actividades de conversación colectiva efectuadas en este proyecto.

De igual forma, aun cuando casi toda la población objetivo maneja y conoce algunos saberes etnobotánicos, no todos quieren o pueden hacer uso de ellos para el manejo de sus síntomas. A esto se suma que el mismo Estado colombiano no reconoce estos conocimientos como válidos para el cuidado de la salud dentro de los planes de desarrollo nacionales, de acuerdo con la información compartida por la comunidad durante el trabajo de campo.

Por otro lado, es importante tener en cuenta el acceso general del departamento a los servicios básicos estatales, con especial atención a la eficiencia y la cobertura del Sistema de Salud [30], puesto que allí encontramos a Bojayá entre los municipios con el acceso más crítico en esta materia [31]. Esto quedó en evidencia en los resultados presentados, ante la enorme cantidad de necesidades insatisfechas en salud individual que fueron develadas por las valoraciones clínicas efectuadas.
No obstante, hay que reconocer que, según documentamos durante nuestro trabajo de campo en conversaciones con autoridades sanitarias departamentales y municipales, durante el tiempo de desarrollo de esta experiencia se dio inicio, desde el Ministerio de Salud y Protección Social, al "Programa de atención psicosocial y salud integral a víctimas" (PAPSIVI), dirigido a los bojayaceños sobrevivientes de la masacre, en cuyo marco algunos habían tenido ya acceso a valoraciones por psicología y algunas áreas de medicina especializada, como oftalmología y ortopedia, entre otras. Sin embargo, el $60 \%$ de quienes participaron en nuestro proyecto negaron haber tenido algún contacto previo con dicho programa, e incluso algunos desconocían su existencia.

La principal limitación de nuestra intervención reseñada derivó precisamente de las dificultades de acceso al territorio habitado por la población con la cual se trabajó. Esto determinó que el proyecto de extensión solidaria universitaria no lograra, en este momento de exploración preliminar e inicio del diagnóstico comunitario, acceder a la totalidad de los supervivientes de la masacre que están identificados por el Comité por los Derechos de las Víctimas de Bojayá, aunque sí se logró llegar a quienes fueron priorizados. Lo anterior hizo recomendable continuar la experiencia mediante fases posteriores, que amplíen la intervención en cobertura y complejidad, según estos resultados preliminares.

\section{Conclusiones}

La exploración preliminar y el diagnóstico comunitario inicial en salud realizado mostraron múltiples dimensiones insatisfechas, relacionadas con el disfrute de derechos fundamentales, como la salud, la vida digna, la educación, el trabajo, entre otros, lo cual requiere ser subsanado a futuro, de modo que queden sentadas las bases de una existencia digna para una comunidad que a la fecha permanece sin recibir reparación debida ante los sufrimientos impuestos por el conflicto armado y por el abandono estatal crónico.

Respecto a las valoraciones individuales efectuadas, los diagnósticos en salud física y mental dejaron en evidencia el persistente requerimiento de una adecuada intervención para el tratamiento de las lesiones resultantes de los hechos vividos durante la masacre del 2002. Llama la atención que, pese a conocerse que las personas sobrevivientes a un estallido de artefacto explosivo improvisado suelen sufrir afectaciones en su sistema auditivo, prácticamente para toda la población abordada nuestra valoración audiológica fue la primera realizada desde el evento traumático. Se evidenció también la falta de ayudas para la audición en una parte importante de las personas.

Del mismo modo, y pese al relato de múltiples proyectos previos de intervención psicosocial que han lle- 
gado a la zona, solo una minoría de las personas abordadas refirió haber tenido en el pasado una valoración clínica individual por el área de salud mental, y en su mayoría no habían recibido intervención terapéutica alguna para afrontar sus síntomas activos. Ello, aunado a que, para la fecha de la evaluación, persistía incertidumbre sobre el paradero e identificación de los restos mortales de los fallecidos en la masacre, que no fueron debidamente recuperados, identificados e inhumados de modo individualizado en su momento, lo que impidió los rituales de duelo propios de la cultura afrocolombiana para despedir a los muertos. Esto dibujó un panorama de enormes necesidades insatisfechas en el campo de la salud mental.

Algo similar ocurría con el dolor crónico sufrido por buena parte de la población, sin manejo apropiado, y con el $33 \%$ de personas que permanecían con esquirlas alojadas en su cuerpo, resultantes de la metralla contenida en el artefacto explosivo improvisado con que fueron atacados, quienes se encuentran sin estudio y manejo para ello, y potencialmente expuestas a los efectos de la liberación crónica de metales pesados dentro de su organismo a partir de dichos cuerpos extraños, como ha sido descrito en series clínicas de militares de otros países que han sufrido lesiones similares [32]. Desde la percepción de los bojayaceños, se ha incrementado la mortalidad por cáncer entre los sobrevivientes de la masacre, y esta es una conexión etiológica, entre otras posibles, que permanece inexplorada.

Pese a la ausencia estatal, los sobrevivientes de la masacre de Bojayá han desplegado su estética vital sin descanso durante todos estos años, afrontando la adversidad a partir de sus conocimientos ancestrales, la unidad comunitaria y de sus organizaciones, y mediante la búsqueda constante de reconocimiento y reparación integral. Donde no ha habido salud pública ni biomedicina, ha acudido la sabiduría ancestral.

Así, se hace evidente la necesidad de entender la dimensión, la posición y la estructura de la racionalidad médica tradicional afrocolombiana en su quehacer cotidiano en favor de la comunidad, y comprender cómo se ha transformado, pensando en que es homóloga a la biomedicina, dadas las condiciones de acceso a ambas por parte de las comunidades habitantes de las riberas y afluentes del río Atrato, pese a la ausencia de reconocimiento estatal para sabedores tradicionales afro, haciéndose necesario apuntalar dicho reconocimiento en busca de su articulación con las redes de servicios alopáticos de salud.

Entre tanto, desde el proyecto también se buscó fomentar la articulación intersectorial e interinstitucional para la atención de necesidades urgentes en salud detectadas en los pobladores de Bojayá víctimas del conflicto, incluso desde la fase preliminar que se reseña, mediante reuniones con representantes de salud departamental y nacional in- volucrados en temas de atención a las víctimas de Bojayá, incluyendo funcionarios del PAPSIVI, tanto en Quibdó como en Bogotá. A futuro es esencial fortalecer el trabajo en red con dichos programas y entidades, para lograr la complementariedad debida en favor de las víctimas.

Es necesario trabajar en la propuesta de un modelo de atención que incorpore las mejores contribuciones que a la salud de la población pueden hacer tanto los conocimientos tradicionales (viendo como ejemplo que los alabaos, como tradición, son cantos que sirven como estrategia cultural de afrontamiento para la población del Chocó biogeográfico) como los propios de la medicina occidental, con el fin de brindar la atención integral que requiere la gente no solo en Bojayá, sino de todos los pueblos de Chocó, teniendo en cuenta las condiciones del acceso a la salud, dadas sus determinantes histórico-sociales.

\section{Agradecimientos}

Al Comité por los Derechos de las Víctimas de Bojayá, a la lideresa comunitaria Delis Palacios, y a las personas sobrevivientes de la masacre del 2 de mayo de 2002 en Bojayá, Chocó, por abrirnos las puertas de sus vidas y de su territorio.

A la antropóloga Ana Carolina Guatáme, por el contacto inicial que brindó con la comunidad y sus invaluables aportes en la construcción de la propuesta inicial del proyecto.

A la psiquiatra Sandra Piñeros, por sus contribuciones en el diseño de ítems de registro para información sobre psicopatología en las evaluaciones de salud mental.

\section{Declaración fuente de financiación}

Trabajo financiado por "Convocatoria Nacional de Extensión Solidaria 2017: Ciencia, tecnología e innovación para la construcción de tejido social", Universidad Nacional de Colombia.

\section{Declaración de conflicto de intereses}

Las personas autoras declaramos no tener conflictos de intereses.

\section{Declaración de responsabilidad}

Declaramos que los puntos de vista expresados son responsabilidad de las personas autoras y no de las instituciones en las que trabajan o de la fuente de financiación.

\section{Declaración de autoría}

Las personas autoras declaramos que todas contribuimos en partes iguales de manera sustancial a la construcción y 
el diseño del artículo, así como a la adquisición, el análisis y la interpretación de datos; del mismo modo, participamos en el diseño del proyecto y su trabajo de campo, aprobamos la versión final a ser publicada, y estamos en capacidad de responder por todos los aspectos del artículo.

\section{Referencias}

1. Colombia, Presidencia de la República. Decreto 893, por el cual se crean los Programas de Desarrollo con Enfoque Territorial - PDET (2017 may. 28).

2. Comité por los Derechos de las Víctimas de Bojayá. Relación de lesionados de la masacre de Bojayá de mayo de 2002 propuestos para atender por el proyecto Laboratorio de Salud Rural Comunidad de Bojayá, Chocó. Quibdó, Chocó: Comité por los Derechos de las Víctimas de Bojayá; 2018.

3. Fals-Borda O. El problema de cómo investigar la realidad para transformarla: por la praxis. Bogotá: Tercer Mundo; 1985.

4. Balcazar FE. Investigación acción participativa (IAP): Aspectos conceptuales y dificultades de implementación. Fundamentos en Humanidades. 2003;1-2(7-8):59-77.

5. Dabas E. Red de redes. Las prácticas de intervención en redes sociales. Buenos Aires: Paidós; 1993.

6. Sluzky C. La red social: frontera de la práctica sistémica. Barcelona: Gedisa; 2006.

7. Talmon N. Single session therapy. Maximizing the effect of the first (and often only) therapeutic encounter. San Francisco: JosseyBass; 1990.

8. Médicos sin Fronteras (MSF). Tres veces víctimas: víctimas de la violencia, el silencio y el abandono. Conflicto armado y salud mental en el departamento de Caquetá, Colombia. Bogotá: MSF; 2010.

9. Fernández Pedraza HF. Comportamiento de la minería legal en el departamento del Chocó 2005-2015 y su impacto en el producto interno bruto, el empleo e infraestructura en la región [monografía de grado]. [Bogotá]: Fundación Universitaria Los Libertadores; 2016.

10. Ruiz G. Perder el lugar: un caso de estudio del desplazamiento en Colombia. European Review of Latin American and Caribbean Studies. 2014;96 55-74. DOI: https://doi.org/10.18352/erlacs.9467

11. Bello M. Bojayá: la culpa de las víctimas y de los victimarios. Desde el Jardín de Freud. 2005;(5):242-55.

12. Bello M, Martín E, Millán C, et al. Bojayá, memoria y río. Violencia política, daño y reparación: Bogotá: Universidad Nacional de Colombia; 2005.

13. Bello MN. La violencia y la masacre en Bojayá: rupturas, daños y recomposiciones desde la perspectiva familiar. En: Puyana Y, Ramírez M-H, editores. Familias, cambios y estrategias. Bogotá: Universidad Nacional de Colombia; 2007. pp. 185-200.

14. Bolívar I, Nieto L. Transformaciones sociales y procesos de intervención "comunidades", "agentes externos" y "conflicto armado" en el caso de Bojayá. Palimpsestus. 2003;(3):68-73.

15. Defensoría del Pueblo de Colombia. Crisis humanitaria en el Chocó. Diagnóstico, valoración y acciones de la Defensoría del Pueblo. Bogotá: Defensoría del Pueblo; 2014.

16. Mosquera C, Tique J. Remembranza de la Escuela César Conto: transformaciones socioculturales, dolor y sufrimiento sobre el emblemático caso de Bojayá. Hallazgos. 2016;13(25):177-97. DoI: https://doi.org/10.15332/s1794-3841.2016.0025.08
17. Quiceno Toro N, Ochoa Sierra M, Villamizar AM. La política del canto y el poder de las alabaoras de Pogue (Bojayá, Chocó). Estudios Políticos. 2017;(51):175-95. Dor: http://dx.doi.org/10.17533/ udea.espo.n51a09

18. Mosquera Mosquera CE, Tique Basto JF. Recuperación de la memoria colectiva sobre la masacre de Bojayá-Chocó, a partir de algunas voces de actores de la Institución Educativa Departamental César Conto [tesis de maestría]. [Bogotá]: Universidad Santo Tomás; 2014.

19. Palacios Mena N. Memoria y violencia: un recorrido por algunas reflexiones y perspectivas. Civilizar: Ciencias Sociales y Humanas. 2017;17(32):209-28. DoI: https://doi.org/10.22518/16578953.827

20. Mosquera CE, Tique Basto JF. Voces desde la escuela de Bojayá en medio del conflicto armado: construcción de su memoria colectiva. Revista de La Universidad de La Salle. 2014;(63):117-34.

21. Jaimes Blanco KE. Crisis de desplazados: Colombia. En: Sociales FdCJy, editor. VIII Congreso de Relaciones Internacionales, 23, 24 y 25 de noviembre de 2016; La Plata: Universidad de La Plata; 2016.

22. Oslender U, Escobar A., et al. Desplazamiento, conflicto y desterritorialización. En: Restrepo E, Rojas A, editores. Conflicto e (in) visibilidad. Retos en los estudios de la gente negra en Colombia. Popayán: Editorial Universidad del Cauca, Organización Internacional para las Migraciones; 2004. pp. 33-138.

23. Ortiz Gutiérrez N. Resistencias pacíficas afrocolombianas. Violencia, desplazamiento forzado y retorno. Bellavista - Bojayá, mayo 2 de 2002 [trabajo de grado]. [Bogotá]: Pontificia Universidad Javeriana; 2011.

24. Alcaldía de Quibdó. Plan municipal de retorno y reubicación integral a las víctimas del conflicto armado 2014-2015. Quibdó: Alcaldía de Quibdó; 2015.

25. Lancheros Ruiz AC, Rincón Ortiz JA. Bojayá: entre el miedo y los medios. En: Mosquera C, Barcelos L, editores. Afro-reparaciones: memorias de la esclavitud y justicia reparativa para negros, afrocolombianos y raizales. Bogotá: Universidad Nacional de Colombia; 2007. pp. 443-66

26. Caicedo LP, Manrique D, et al. Desplazamiento y retorno. Balance de una política. Libro 2. Espirales del desplazamiento. El retorno a Bojayá, Chocó. Bogotá: ILSA; 2006.

27. Chenut Correa P, Prado MO. Reasentamiento tras el desplazamiento forzado: dos comunidades étnicas en Colombia. Iztapalapa. Revista de Ciencias Sociales y Humanidades. 2014;35(76):105-37. DoI: https:// doi.org/10.28928/ri/762014/atc4/chenutcorreap/ocampopradom

28. Rengifo Carpintero JA, Díaz Caicedo CH. El canto como mecanismo de reparación en las comunidades negras, víctimas de la violencia, del Pacífico colombiano: entretejiéndose con el Grupo Integración Pacífico. Antropología Experimental. 2018;(18):15164. https://doi.org/10.17561/rae.v0i18.3452

29. Pinilla Bahamón AM. Alabaos y conflicto armado en el Chocó: noticias de supervivencia y reinvención. Encuentros. 2017;15(3):15269. DoI: http://dx.doi.org/10.15665/re.v15i3.1096

30. Martínez Mora JA. Monitoreo al sistema de salud en el departamento del Chocó: 1998-2004. Ensayos sobre economía regional. Bogotá: Banco de la República; 2005.

31. Moscoso M, Restrepo J. Capacidades institucionales en materia económica para municipios en Colombia. Documentos de Trabajo CERAC; 2013.

32. Gaitens J, Dorsey C, McDiarmid M. Using a public health registry to conduct medical surveillance: The case of toxic embedded fragments in U.S. military veterans. Eur J Oncol. 2010;15(2):77-89. 